Revue scientifique sur la conception et l'aménagement de l'espace

$10 \mid 2014$

Le paysage à l'épreuve de la transition énergétique

\title{
Réseau électrique et réseau écologique : contraintes et opportunités dans un paysage fragmenté (Nord-Pas-de-Calais)
}

Electric Grid and Ecological Network: Constraints and Opportunities in a Fragmented Landscape (Nord-Pas de Calais)

Guillaume Schmitt, Magalie Franchomme et Christelle Hinnewinkel

\section{OpenEdition}

\section{Journals}

Édition électronique

URL : http://journals.openedition.org/paysage/11751

DOI : 10.4000/paysage. 11751

ISSN : 1969-6124

Éditeur :

École nationale supérieure du paysage de Versailles-Marseille, Institut national des sciences appliquées Centre Val de Loire - École de la nature et du paysage, École nationale supérieure d'architecture et de paysage de Bordeaux, École nationale supérieure d'architecture et de paysage de Lille, Agrocampus Angers

Référence électronique

Guillaume Schmitt, Magalie Franchomme et Christelle Hinnewinkel, « Réseau électrique et réseau écologique : contraintes et opportunités dans un paysage fragmenté (Nord-Pas-de-Calais) », Projets de paysage [En ligne], 10 | 2014, mis en ligne le 31 août 2014, consulté le 15 octobre 2020. URL : http:// journals.openedition.org/paysage/11751 ; DOI : https://doi.org/10.4000/paysage.11751

Ce document a été généré automatiquement le 15 octobre 2020.

Projets de paysage 


\title{
Réseau électrique et réseau écologique : contraintes et opportunités dans un paysage fragmenté (Nord-Pas-de-Calais)
}

\author{
Electric Grid and Ecological Network: Constraints and Opportunities in a \\ Fragmented Landscape (Nord-Pas de Calais)
}

Guillaume Schmitt, Magalie Franchomme et Christelle Hinnewinkel

1 Il est aujourd'hui généralement admis que le paysage est ce que l'on voit d'un espace et ne se limite pas à l'agencement matériel de ce dernier. Cependant, comme nous le suggère la métaphore de la Tulipe utilisée par Lassus dans la préface de l'ouvrage d'Henri et Odile Décamps (2004, p. 9 sq.), un regard sur l'organisation physique du paysage, tel que le propose l'écologie du paysage, ouvre des perspectives dans la manière de le regarder, et donc d'aménager les territoires. Cet article propose d'analyser la relation complexe entre deux éléments du paysage que sont le réseau électrique et le réseau écologique pour mieux comprendre comment agir sur le premier afin de maintenir ou de renforcer le second. La région Nord-Pas-de-Calais sert de terrain à cette étude. Le maintien et la création d'un réseau écologique sont, pour ce territoire très urbanisé, des enjeux majeurs pour les pouvoirs publics depuis les années 1990 (CAUE Nord, 1993).

2 Contribuant à la réduction et à la dégradation des habitats naturels, l'urbanisation est l'une des principales causes de déclin de la biodiversité en France (Sainteny et al., 2012). Les grandes infrastructures linéaires de transport (ILT) ${ }^{1}$ concourent à la fragmentation des habitats et au cloisonnement des espèces (Fahrig, 2003; Jaeger et Fahrig, 2004). Elles limitent, en outre, les mouvements de populations animales et leurs échanges génétiques par des effets de barrière.

3 La mise en place de la Trame verte et bleue (TVB), ainsi que prévue par la loi Engagement national pour l'environnement ${ }^{2}$, doit permettre de «lutter efficacement 
contre l'érosion de la biodiversité ». Conçue comme un outil d'aménagement du territoire, la TVB doit permettre d'intervenir sur des territoires fragmentés via le maintien ou la restauration des continuités écologiques. Or, dans un contexte de forte densité démographique et d'une fragmentation importante des paysages, elle pourra difficilement être déployée sans une réflexion sur les potentialités que peut offrir le foncier des ILT.

4 Notre étude porte sur les infrastructures, dont Réseau de transport d'électricité (RTE) ${ }^{3}$ a la charge dans le Nord-Pas-de-Calais, et s'inscrit dans un projet de recherche financé par la Fondation de recherche pour la biodiversité sur les réseaux d'acteurs de la biodiversitét ${ }^{4}$. Ce projet a mis en évidence le souhait de plusieurs collectivités locales d'intégrer les ITL à leur TVB. Ainsi, nous sommes partis du postulat que les ILT, qu'ils s'agissent des infrastructures elles-mêmes, des dépendances vertes, des zones d'emprunts ou des délaissés, pouvaient se concevoir non plus principalement comme des éléments fragmentant aux externalités négatives, mais aussi comme d'éventuelles opportunités à saisir, notamment foncières, dans la mise en œuvre des TVB. Le patrimoine foncier de RTE a, comparativement aux autres ILT, une faible emprise au sol, mais son réseau impose, en raison du surplomb des lignes, des contraintes de constructibilité, de hauteur et de type de couvert végétal.

5 Avant de présenter la démarche d'analyse mise en place dans le champ de l'écologie du paysage, les spécificités du réseau de l'opérateur et du terrain d'étude sont présentées afin de comprendre les logiques actuelles au regard de la préservation de la biodiversité. Les éléments linéaires et les emprises ponctuelles de RTE sont ensuite croisés à la TVB adoptée en 2006 (schéma régional d'aménagement et de développement du territoire), en vue de qualifier les opportunités de la mise en synergie de ces deux réseaux ${ }^{5}$.

\section{Des connexions établies : réseau électrique et réseau écologique}

6 Le contexte législatif (loi ENE, étude d'impact, contrat de performance, mesures compensatoires...) a amené les opérateurs d'ILT à intégrer la biodiversité dans leur stratégie de développement. Progressivement, des actions ponctuelles et/ou pérennes, allant du plan de gestion au financement de projets de recherche, sont mises en place. Ainsi, le regard porté sur les ILT et leurs effets, que ce soit sur le paysage ou plus généralement sur l'environnement, a depuis peu considérablement évolué jusqu'à leur attribuer un rôle dans la mise en place de la TVB. La région Nord-Pas-de-Calais illustre cette évolution, puisque la prise en compte des lignes électriques a connu une évolution sémantique notable. Ces dernières, classées comme «facteur de dégradation » en 2005 (Hendoux, 2005, p. 68) sont désormais des objets à intégrer dans le Schéma régional de cohérence écologique (SRCE) «dans un souci de cohérence nationale ( (préfet Région Nord-Pas-de-Calais, 2012a, p. 214).

\section{Vers un nouveau rapport entre infrastructures linéaires de transport et biodiversité ?}

7 Les effets des ILT ont fait l'objet de nombreuses études. Van Der Zande et al. (1980), Andrews (1990), Spellerberg (1998), par exemple, se sont penchés sur l'incidence des 
routes sur la distribution et la mortalité de plusieurs espèces de mammifères, d'oiseaux, de reptiles et d'amphibiens. Des auteurs ont démontré une corrélation positive entre les lignes électriques et la "surmortalité » des certaines espèces de corvidés et de rapaces en raison des risques de collision ou, dans certaines configurations, d'électrocution qui leur sont associés (Rubolini et al., 2005 ; Kabouche et al., 2006). En 2010, à partir d'une analyse statistique de 49 études, Benitez-Lopez et al. ont mis en évidence que la proximité des ILT influence aussi bien la densité de la population (réduction du nombre d'individus) de plus de 230 espèces de mammifères et d'oiseaux que leur mouvement (stratégie d'évitement). En outre, les populations de mammifères sont affectées sur des distances beaucoup plus grandes que celles des oiseaux $(5 \mathrm{~km}$ contre $1 \mathrm{~km}$ pour les oiseaux) avec des variations selon les taxons et le type d'habitat. En effet, les perturbations semblent plus importantes dans des zones ouvertes que dans les zones boisées. Dans le même esprit, Clauzel et al. (2013) ont démontré des effets négatifs majeurs dans les premiers $500 \mathrm{~m}$ des lignes ferroviaires à grande vitesse sur la distribution de la rainette verte.

8 Une synthèse des impacts écologiques négatifs, directs ou indirects, des ILT a été publiée par Alsace Nature en 2008. La perte d'habitat et la pollution sont à ajouter aux précédentes perturbations citées. L'étude qualifie de "points de conflits" les intersections entre les ILT et les principales continuités écologiques, qu'elles soient existantes ou à recréer (ibid., p. 31), et propose des actions de gestion, telles que l'ensemencement de prairies fleuries ou encore la plantation arbustive sous les lignes ou au niveau des pylônes. En effet, le maintien d'un couvert arbustif permet de réduire la perte de biodiversité et d'éviter la propagation des espèces invasives (Clarke et White, 2008). La gestion de la richesse floristique et de la structure de la végétation, notamment par des défrichages ou par des fauches fréquentes, peut aussi avoir des effets positifs sur les populations de papillons (Berg et al., 2013).

9 Les effets positifs des ILT sur la biodiversité sont aussi de plus en plus mis en évidence. Par exemple, Andrews (1990) cite plusieurs publications qui montrent que les milieux qui longent les ILT peuvent constituer des habitats favorables à l'avifaune ou servir de corridor écologique (Meehan et Haas, 1997 ; Askins et al., 2012). Une étude récente conforte ce point de vue en proposant une synthèse de 92 publications qui montre les effets positifs des ILT sur les oiseaux (Morelli et al., 2014). Cette synthèse souligne que les accotements routiers et les pieds de pylônes peuvent offrir des habitats d'alimentation, les lignes électriques servent de perchoirs utiles, notamment en période de chasse et des nids sont construits sur des pylônes.

10 Par conséquent, le comité opérationnel TVB ne caractérise pas les intersections entre continuités écologiques et ILT uniquement comme des "points de conflits", mais également comme des supports potentiels de biodiversité et préconise de «prendre en compte la biodiversité dans les infrastructures linéaires de transport » et « d'intégrer la Trame verte et bleue dans les réseaux d'infrastructures existantes » (Allag-Dhuisme et al., 2010, p. 23 sq.). Des méthodes de prise en compte de la biodiversité, notamment dans le choix d'un tracé d'infrastructure, vont alors être développées et devenir de plus en plus complexes (Vandevelde, 2013 ; Vanpeene-Bruhier et al., 2013).

11 Pour les opérateurs en charge des ILT, la prise en compte du paysage et de la biodiversité semble avoir considérablement évolué ces dernières années. Cela se traduit par la signature de plusieurs conventions de partenariat, à l'instar de celles signées par RTE. En 2008, RTE s'engage avec l'Association des maires de France à une meilleure 
intégration des transports électriques dans les programmes d'aménagement du territoire et sur des actions communes en faveur du développement durable. Parmi les thèmes ciblés figure la protection de la biodiversité et des paysages (RTE, AMF, 2008, p. 5). La même année, l'opérateur signe une convention avec la Fédération nationale des chasseurs qui s'inscrit dans le cadre de l'engagement national d'enrayer la perte de biodiversité. Dans cette convention RTE affirme "être attentive à la gestion de ses emprises ainsi qu'à ses pratiques d'élagage et de travaux de maintenance » (RTE, FNC, 2008, p. 8). RTE réaffirme son implication dans la TVB et son souhait d'identifier et de valoriser le développement d'une biodiversité singulière dans les couloirs de lignes via le renouvellement, en 2010, de son partenariat avec l'Association nationale des élus de la montagne (RTE, ANEM, 2013, p. 3).

Enfin, en 2011, les sept plus importants gestionnaires d'infrastructures du territoire

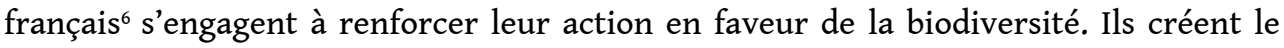
Club infrastructures linéaires et biodiversité (CILB) et signent à cette occasion une charte dont l'objectif est de concilier leurs activités avec les enjeux de préservation de la biodiversité. En 2012, le CILB adhère à la stratégie nationale pour la biodiversité. Ainsi, l'engagement des gestionnaires, mis en perspective avec les objectifs du Grenelle de l'environnement, incite à questionner les éventuelles contributions des ILT à la préservation et à la remise en bon état des continuités écologiques.

\section{Choix du terrain d'expérimentation}

13 S'intéresser aux relations entre le réseau RTE et la TVB requiert un terrain d'étude qui, s'il n'a pas vocation à être représentatif de l'ensemble des configurations territoriales, se caractérise tout au moins par la diversité des connexions possibles (Konga et al., 2010). Dans cette perspective, trois critères sont apparus comme incontournables et complémentaires. Tout d'abord, les démarches relatives à la TVB doivent être éprouvées et anciennes pour assurer une stabilité dans le temps et dans l'espace des périmètres identifiés. Ensuite, le réseau électrique doit offrir des configurations variées et un vaste champ d'expérimentation. Enfin, nous partons de l'hypothèse que dans un territoire fortement fragmenté, des actions, même ponctuelles, le long du réseau RTE auront des effets en matière de préservation de la biodiversité. La région Nord-Pas-deCalais regroupe l'ensemble de ces critères.

Dès 1993, la région Nord-Pas-de-Calais lance avec les services de l'État le projet Ecotone, dont l'objectif est de construire une "Trame écologique régionale » (CAUE Nord, 1993). Par la suite, d'autres initiatives émergent, à l'exemple du classeur "Trame verte » de 1995 ou des "contrats de corridor biologique». En 1999, le Schéma de services collectifs des espaces naturels et ruraux (SSCENR) ${ }^{7}$ répertorie les grandes liaisons biologiques et propose de "planifier la mise en place de corridors biologiques entre tous les secteurs à biodiversité » (Diren Nord-Pas-de-Calais, 1999, p. 135). Enfin, le Schéma régional d'aménagement et de développement du territoire (SRADT) intègre en 2006 un volet spécifique sur la TVB et des atlas à l'échelle régionale et intercommunale (Région Nord-Pas-de-Calais, 2006). Le projet de SRCE est dénommé SRCE-TVB, afin d'entériner l'évidente filiation.

La précocité de ces démarches s'explique en grande partie par les caractéristiques de la région Nord-Pas-de-Calais. En effet, les espaces naturels sont parmi les plus fragmentés de France ${ }^{8}$. Le territoire est composé de quelque 4 millions de taches ${ }^{9}$, dont 15000 sont 
isolées par les ILT (Biotope, 2008). En considérant également l'armature urbaine et la densité démographique, les orientations nationales pourront difficilement être atteintes sans une réflexion sur les potentialités des réseaux de transports linéaires, dont le réseau électrique. Dans le projet actuel, le réseau électrique est identifié comme élément fragmentant, mais aussi support potentiel à la TVB « dès lors qu'il existe des données fiables sur la qualité écologique » des espaces concernés (préfet du Nord-Pasde-Calais et région Nord-Pas-de-Calais, 2012a, p. 214). Comparativement à la région Rhône-Alpes, le Nord-Pas-de-Calais présente une longueur de réseau assez faible (3 $225 \mathrm{~km}$ ), mais particulièrement dense, avec en moyenne 250 mètres de fils par km².

Figure 1. Densité du réseau haute tension (HT)et très haute tension (THT) en France par région

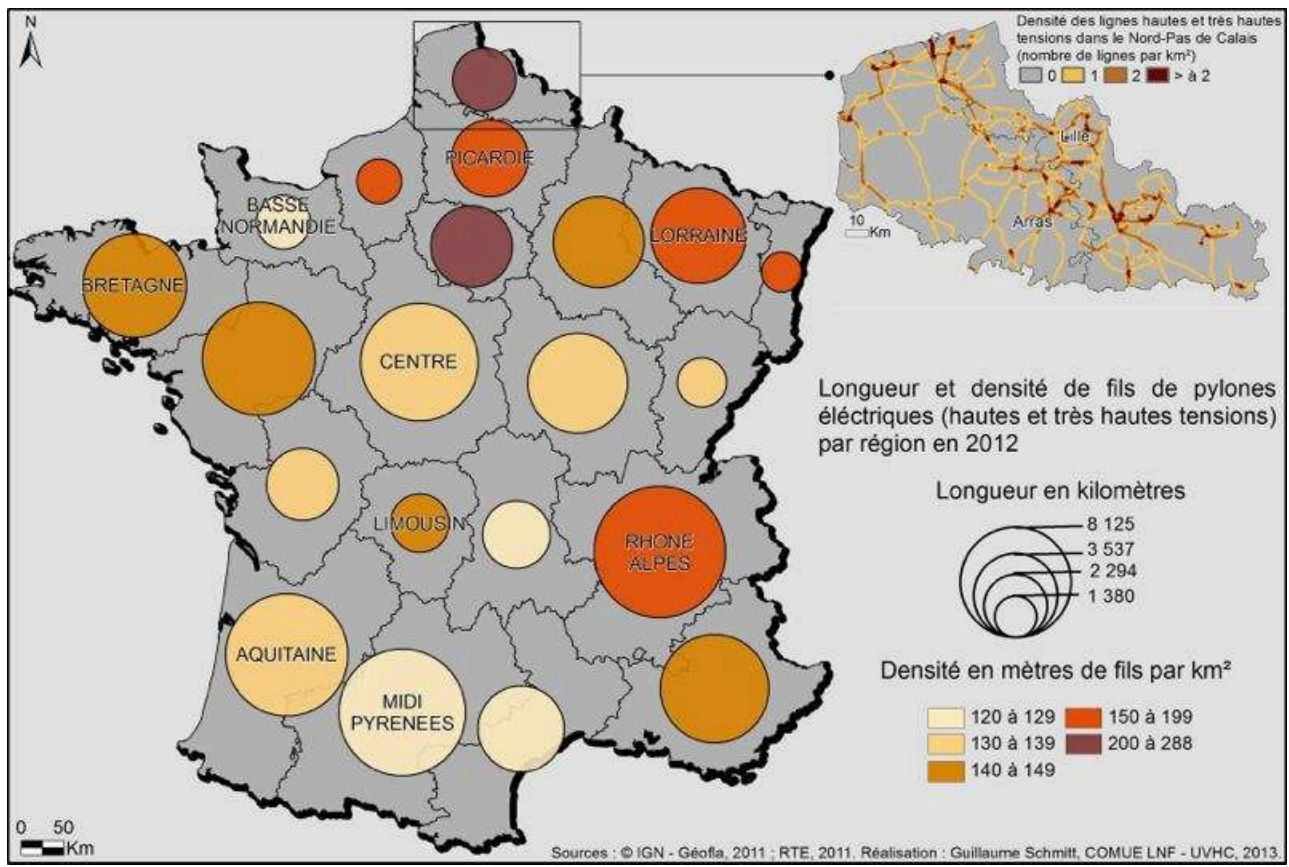

Il n'est donc pas rare que plusieurs lignes à haute et à très haute tension (HT et THT) se jouxtent sur moins d'un kilomètre, notamment à proximité des grandes agglomérations. De plus, quelque 12000 pylônes et 175 postes de transformation jalonnent l'espace régional. Cette forte densité des éléments du réseau HT et THT amène d'ailleurs les acteurs en charge de la TVB à les considérer comme une des caractéristiques du paysage en plaine maritime picarde (p. 161) ou dans le Haut-Artois (p. 169).

Plus généralement, le rôle des éléments du paysage sur la diversité biologique est largement étudié depuis les années 1980 (Forman et Godron, 1986). Arrif et al. (2011) ont d'ailleurs souligné les différentes ramifications de la généalogie de la notion de Trame verte (écologie du paysage, aménagement de l'espace, urbanisme, géographie et sociologie), ainsi que leurs relations récentes, notamment par l'émergence de la notion de paysage urbain. À l'échelle locale ${ }^{10}$, la référence au paysage est ainsi récurrente (Bourget et Le Dû-Blayo, 2010), d'autant plus qu'il contribue au rapprochement « des formes concrètes d'un territoire, des logiques de développement territorial et des perceptions des acteurs» (Cormier et Carcaud, 2009). Le Nord-Pas-de-Calais ne fait pas office d'exception puisque dans le document cadre du SRADT la « mise en œuvre de la Trame verte et bleue » est décrite comme « une politique ambitieuse du paysage » et un 
moyen de «reconstituer [...] une infrastructure naturelle plurifonctionnelle (écologique, paysagère, ludique et source d'activités) » (Région Nord-Pas-de-Calais, 2006, p. 104).

Pour qualifier les connexions entre le réseau électrique et la TVB, nous avons cherché à identifier les types d'utilisation du sol sous l'influence des réseaux HT et THT et à estimer son importance dans le paysage vu.

\section{Le réseau électrique : une caractéristique du paysage}

19 L'implantation de nouveaux réseaux électriques fait l'objet d'études d'impact, de concertations et aussi de conflits (Medd et Ademe, 2005 ; Billet, 2006 ; Denolle, 2011) ${ }^{11}$. Après la production d'électricité nucléaire, Guillaume Bouvier (2003) considère que le paysage est le second enjeu géopolitique de la distribution d'énergie électrique en France. Cependant, notre propos ici n'est pas d'apporter une contribution aux problématiques sociales. Nous cherchons avant tout à qualifier et à quantifier la nature de l'utilisation du sol influencée par le réseau HT et THT pour pouvoir qualifier les connexions entre ce dernier et la TVB.

\section{Modélisation de l'emplacement du réseau électrique dans le paysage : des points et des lignes grevant des surfaces}

La constitution d'une base de données du réseau électrique se confronte à deux écueils. Le premier est relatif à la représentation cartographique des éléments de ce réseau au sein d'un système d'information géographique (SIG). Ces éléments sont traduits sous une forme géométrique simple, soit en point, soit en ligne, ce qui limite l'analyse statistique résultant de la confrontation aux référentiels d'utilisation du sol existants (calcul de superficie, taux de recouvrement, etc.). Or, ne pas prendre en compte les contraintes de constructibilité, la hauteur autorisée du couvert végétal, l'emprise au sol des pylônes reviendrait à négliger leurs rôles sur l'utilisation du sol alentour.

21 Le second est l'inadéquation des principales bases de données géographiques à l'analyse envisagée. Par exemple, dans le référentiel géographique à grande échelle de l'IGN, la hauteur des pylônes et la largeur des servitudes d'utilité publique ne sont que très rarement renseignées. Ainsi plusieurs méthodes ont été compilées pour constituer un jeu de données.

Dans un premier temps, le tracé des éléments et les informations thématiques générales (voltage, etc.) ont été extraits des bases de données de l'IGN et de RTE. La largeur des nappes de câbles et la hauteur des pylônes ont en revanche été estimées par photo-interprétation, sur la base d'un échantillonnage aléatoire. Plus de 250 pylônes et 150 kilomètres de lignes ont fait l'objet de mesure.

Dans un second temps, nous avons cherché à identifier les contraintes et les effets des ouvrages électriques sur l'utilisation du sol. La consultation des guides techniques et de plans locaux d'urbanisme (PLU) ${ }^{12}$ a permis de déduire les périmètres contraints par la présence des éléments du réseau, en raison des servitudes d'ancrage, d'appui, d'élagage et d'abattage d'arbres ${ }^{13}$ (EDF et GDF, 2000; RTE, 2005). Cette étape a permis de cartographier le réseau électrique non plus sous forme ponctuelle ou linéaire mais sous la forme de surfaces. 

évidence les spécificités du réseau HT et THT.

Figure 2 : Utilisation du sol le long des lignes HT et THT dans le Nord-Pas-de-Calais

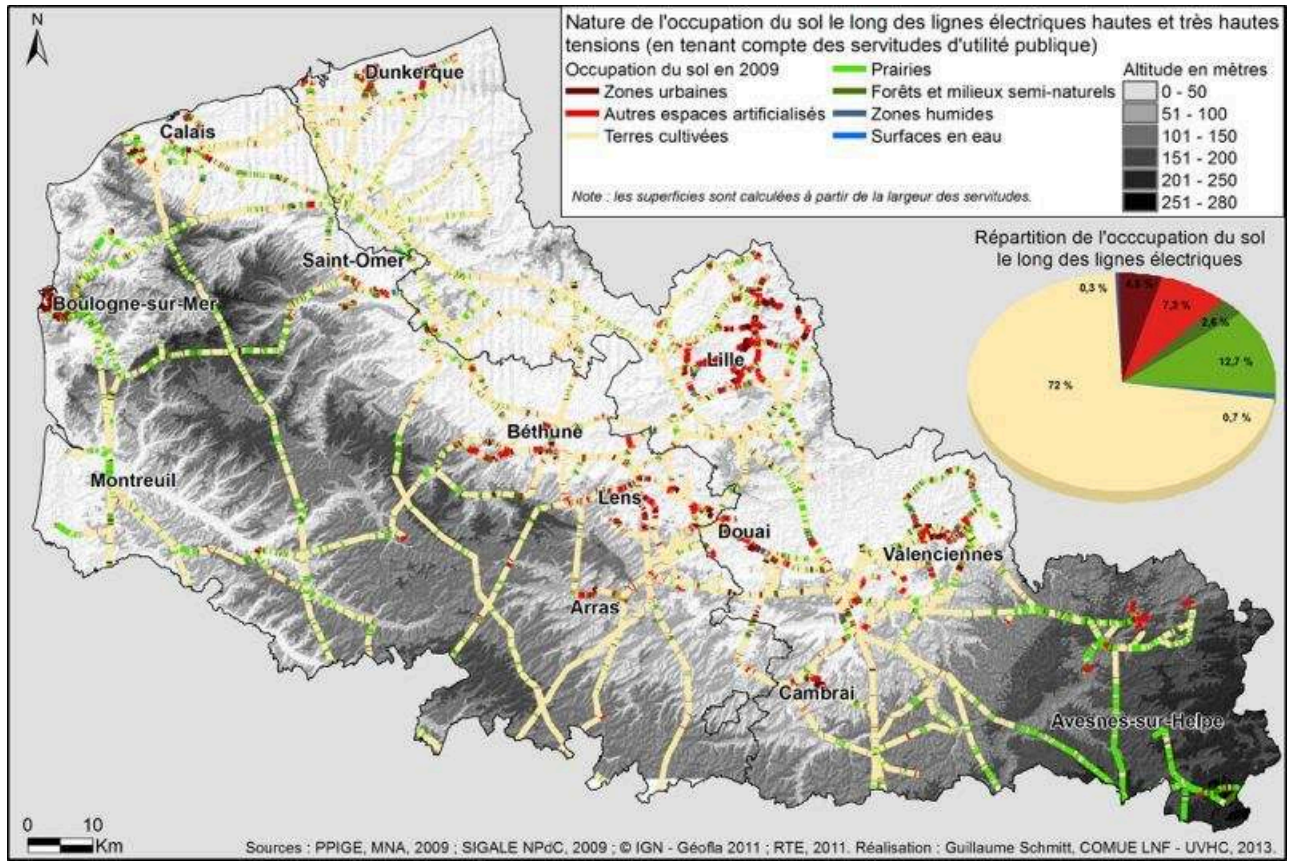

Alors que le Nord-Pas-de-Calais est une région très urbanisée (plus de $10 \%$ de sa superficie), les lignes HT et THT surplombent rarement les secteurs urbains (seulement $4,5 \%$ de l'ensemble du réseau). En revanche, elles sont logiquement plus présentes en zones industrielles ou commerciales ${ }^{15}$, puisque ces dernières nécessitent pour leur fonctionnement un raccordement à un voltage élevé et surplombent majoritairement les terres cultivées (72\%).

Les forêts et milieux semi-naturels sont moins affectés par le réseau électrique $(2,6 \%)$ que les prairies $(12,7 \%)$. Les proportions sont équivalentes pour les pylônes. Ainsi, il apparaît que le réseau électrique traverse principalement des secteurs où le potentiel de biodiversité est généralement qualifié d'ordinaire, faible ou moyen, que ce soit pour la faune ou la flore (Lienard et Clergeau, 2011 ; Vimal et Mathevet, 2011).

Les surfaces grevées, surtout celles des pylônes, sont depuis peu étudiées en tant que secteur de moindre intensité des pratiques culturales et de relais potentiels vers des habitats plus propices pour la faune et la flore. Les servitudes attenantes sont également envisagées comme des moyens de limiter la fermeture des paysages (Le Floch et al., 2005) et de maintenir des « espèces végétales disparues ou menacées [...] qui affectionnent les espaces ouverts » (Valet, 2011, p.3). Deux récentes études sur les 
emprises au sol des pylônes électriques et la diversité floristique en zone de culture ont été menées entre 2009 et 2012. La première est un inventaire floristique mené par le Cemagref et le Muséum national d'histoire naturelle de Paris, où la situation sous les pylônes et celle dans l'espace de culture avoisinant sont comparées ${ }^{16}$. La seconde se focalise sur 150 pylônes dans le Nord-Pas-de-Calais, notamment choisis par la Fédération régionale de la chasse, RTE et le groupe ornithologique et naturaliste du Nord $^{17}$.

Suivant les orientations nationales du Comité opérationnel Trame verte et Bleue (COMOP-TVB), les acteurs régionaux et locaux réfléchissent à l'intégration des pylônes aux différents projets de TVB afin de créer des îlots-refuges pour la faune et ainsi restaurer des corridors écologiques « en pas japonais ». Dans cette optique et parce que la distance entre chaque " pas » est une variable discriminante selon les espèces, nous avons cherché à identifier des secteurs favorables à la création de tels corridors en retenant le critère de la covisibilité. Un modèle numérique de terrain et un modèle de surface fondé sur la cartographie de l'utilisation du sol ont été utilisés afin de prendre en compte la hauteur des ouvrages, le relief des lieux d'implantation, la distance et les obstacles présents entre les pylônes. L'analyse de covisibilité est ici utilisée pour estimer la répartition des pylônes et permet d'identifier, dans l'absolu, les portions du territoire pour lesquelles l'aménagement de corridors « en pas japonais » via les pieds de pylônes pourrait potentiellement être pertinent. Néanmoins, pour déterminer la fonctionnalité des corridors ainsi générés, il conviendrait d'y adosser une analyse de connectivité écologique fonctionnelle. Faute de données naturalistes suffisamment fines, cette analyse n'a pu être effectuée.

Figure 3. Covisibilité estimée des pylônes électriques à HT et à THT dans le Nord-Pas-de-Calais

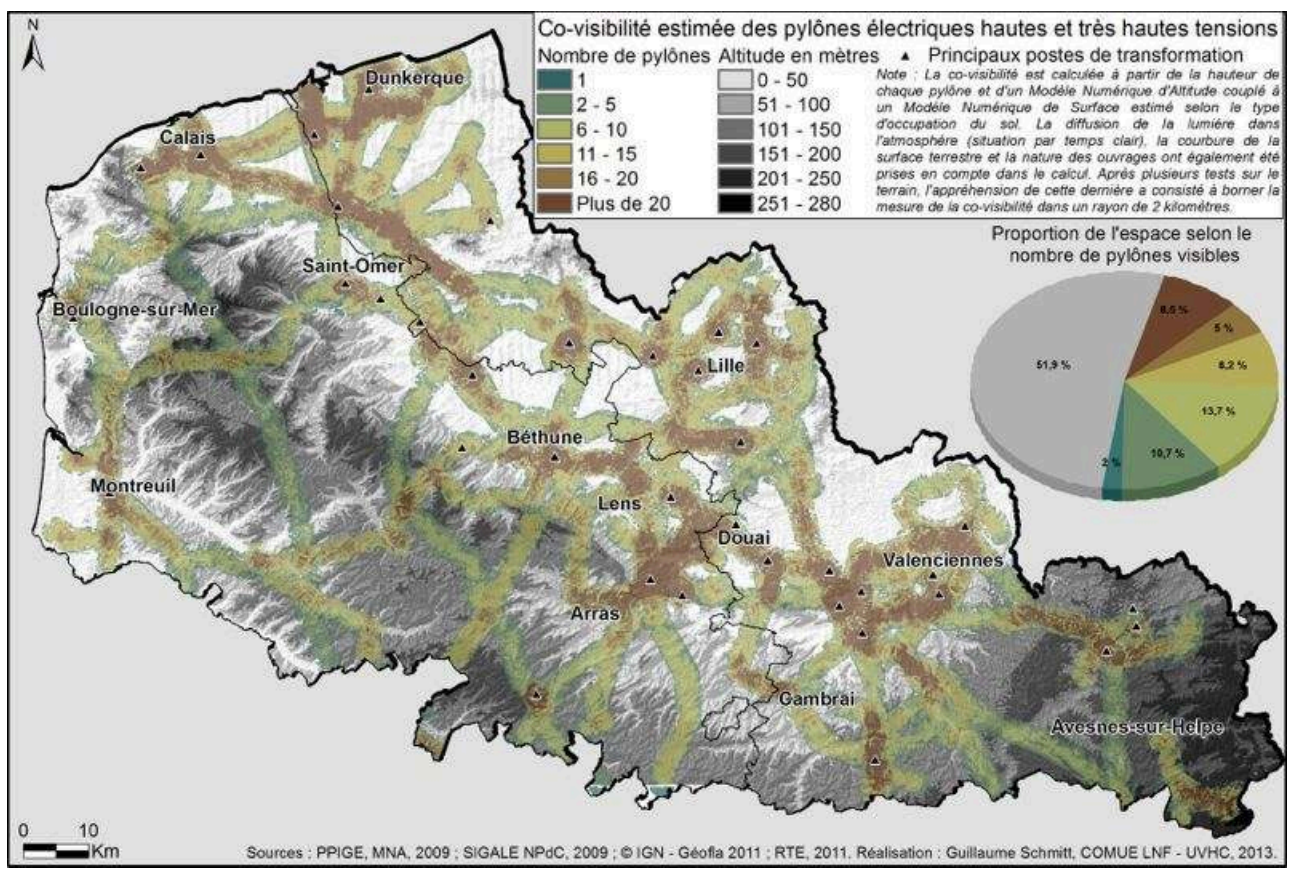

30 La forte covisibilité entre les pylônes s'explique, d'une part, par le grand nombre de pylônes dans la région et, d'autre part, par la faiblesse des pentes. De plus, ces ouvrages apparaissent comme une des caractéristiques du paysage, puisque, sur environ un cinquième de l'espace régional, il est possible de voir plus de 10 pylônes. Ceci explique 
sans doute l'ambition des acteurs du territoire de les mobiliser comme des opportunités dans le SRCE-TVB. Mais peuvent-ils pour autant contribuer réellement au maintien ou au renforcement des continuités écologiques terrestres?

\section{Le réseau électrique peut-il contribuer à la TVB ?}

31 Pour répondre à cette question, nous avons étudié le niveau de recouvrement de surface entre le réseau électrique et la TVB et mené une simulation des effets sur la connectivité structurelle d'un changement de l'utilisation du sol sous les pylônes.

\section{Une corrélation spatiale significative entre le réseau électrique et la TVB}

La comparaison entre le réseau électrique et le réseau écologique repose sur le schéma de TVB adopté en 2006. Les principes de l'écologie du paysage restent inchangés entre ce dernier et le SRCE :

«La désignation des réservoirs de biodiversité, des espaces naturels relais et des corridors écologiques n'a pas fondamentalement changé dans ses grands principes. » (Préfet du Nord-Pas-de-Calais et Région Nord-Pas-de-Calais, 2012b, p. 48.)

Ce schéma identifie une TVB constituée de cœurs de nature, d'espaces naturels relais, d'espaces à renaturer et de corridors ${ }^{18}$.

L'étude du recouvrement de surface entre réseau électrique et TVB met en évidence une corrélation positive et significative d'ensemble qui est toutefois moins prononcée pour la TVB que pour le réseau électrique.

Figure 4. Corrélations spatiales entre le réseau HT et THT et la TVB du Nord-Pas-de-Calais de 2006

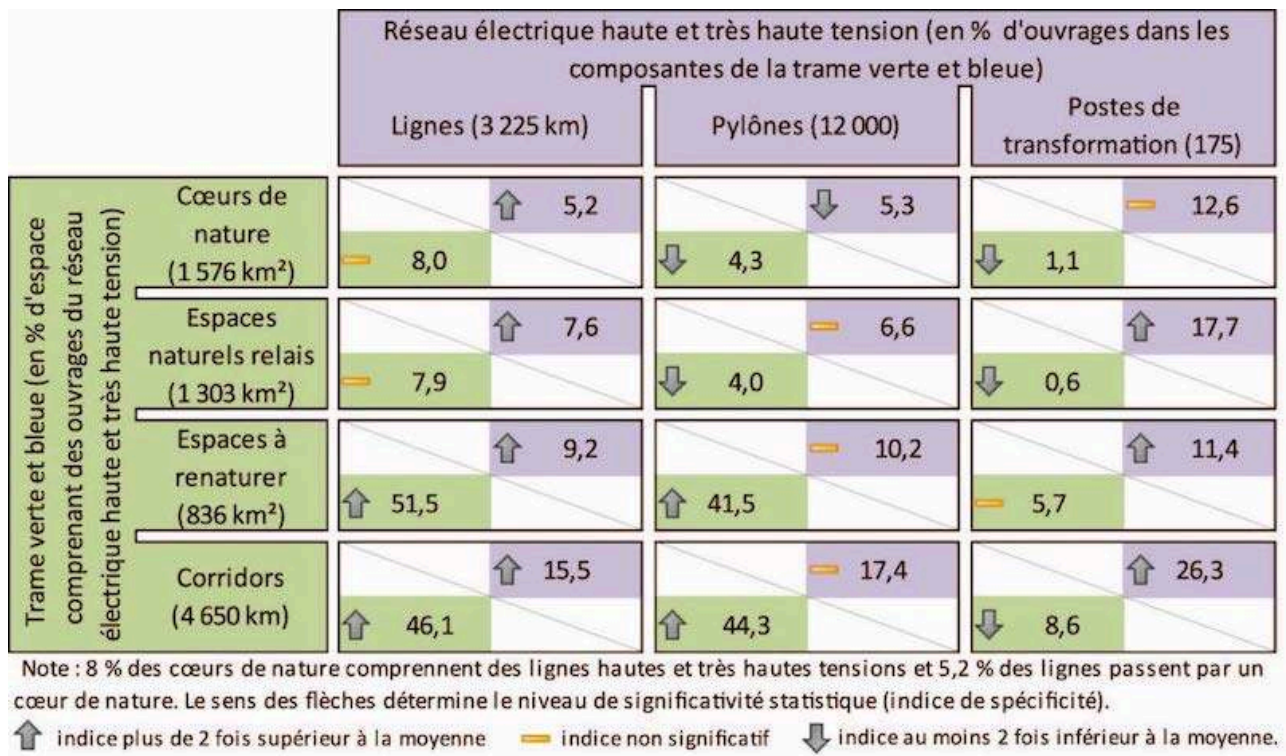

Deux idées principales se dégagent de la matrice de corrélation. D'une part, une partie importante du réseau électrique du Nord-Pas-de-Calais traverse le réseau de la TVB : un tiers des lignes électriques surplombent un élément de la TVB et plus de la moitié des 
postes de transformation y sont situés. D'autre part, de nombreux corridors et des espaces à renaturer sont traversés par le réseau électrique: environ la moitié des espaces à renaturer sont survolés par des lignes électriques ; et des pylônes électriques sont présents dans $44 \%$ des tracés de corridors et $41 \%$ des espaces à renaturer.

Les pieds de pylônes étant sous la gestion de RTE, l'opérateur pourrait mettre en œuvre des actions de génie écologique en faveur de la faune pour contribuer au renforcement des continuités écologiques. Une gestion écologique des pieds de pylônes consiste généralement en la modification de l'utilisation du sol sous les pylônes pour créer un habitat de substitution destiné à une faune particulière qui a besoin de grands espaces pour se maintenir. Afin de mieux apprécier le rôle que peuvent potentiellement jouer des actions de génie écologique sous les pylônes, nous avons cherché à tester les effets sur les corridors écologiques d'une modification de l'utilisation du sol située sous les pylônes.

\section{Les composantes du réseau, des leviers pour favoriser les continuités écologiques?}

La mosaïque paysagère est tout d'abord modélisée selon sa perméabilité au déplacement d'une espèce cible (Hirzel et al., 2001). La présence, l'absence et l'abondance relative d'une espèce par types d'habitat servent généralement de référence (Calabrese et Fagan 2004; Cemagref, 2010). Ces variables sont estimées par comptage échantillonné. Les mesures sont ensuite transposées en valeur de résistance dans les cartographies d'utilisation du sol. L'article de Kindlmann et Burel en 2008 fournit un panorama de publications sur la perméabilité de la mosaïque paysagère. $\mathrm{Ne}$ disposant pas de comptages, nous avons établi des analogies avec les études citées, afin de bâtir une modélisation en fonction des déplacements d'un mammifère commun du Nord de la France. De plus, l'étalonnage des valeurs de résistance s'obtient «par tâtonnement", puisque leur détermination ne répond pas à des critères établis (Foltête, 2006, p. 170). Après plusieurs tests, la mosaïque paysagère est modélisée selon six niveaux de perméabilité croissants : zones infranchissables, zones à éviter, zones de passages occasionnels, habitats de substitution, habitats propices à la reproduction et habitats assurant la reproduction et un taux de survie élevé.

À partir de la modélisation de la perméabilité sur l'ensemble du Nord-Pas-de-Calais, nous avons ensuite simulé le déplacement de l'espèce cible à partir de deux méthodes complémentaires. Pour identifier le trajet de moindre résistance entre deux cœurs de nature, nous avons utilisé la méthode exposée par Caloz et Colet (2011). Elle a la particularité d'être sensible à la résolution spatiale des données mobilisées et aux valeurs de degré de résistance (Broquet et al., 2006). Elle répond donc partiellement à notre problématique où il est question de modification de l'utilisation du sol sur de faibles emprises spatiales (pylônes). Pour identifier un couloir de circulation plus large correspondant davantage aux déplacements de l'espèce cible, les critères suivants ont été retenus : une largeur comprise entre un mouvement optimum (le plus court en distance euclidienne et en distance coût) et un déplacement incluant la divagation (Beier et al., 2008). Des contraintes de franchissement (autoroute, canal, etc.) et de distances minimales aux zones urbaines denses sont également intégrées à la simulation. 
Figure 5. Simulation des effets de création d'habitats de substitution sous des pylônes électriques HT et THT sur la connectivité écologique structurelle et le coût de déplacement d'un mammifère entre deux cœurs de nature de la TVB du Nord-Pas-de-Calais

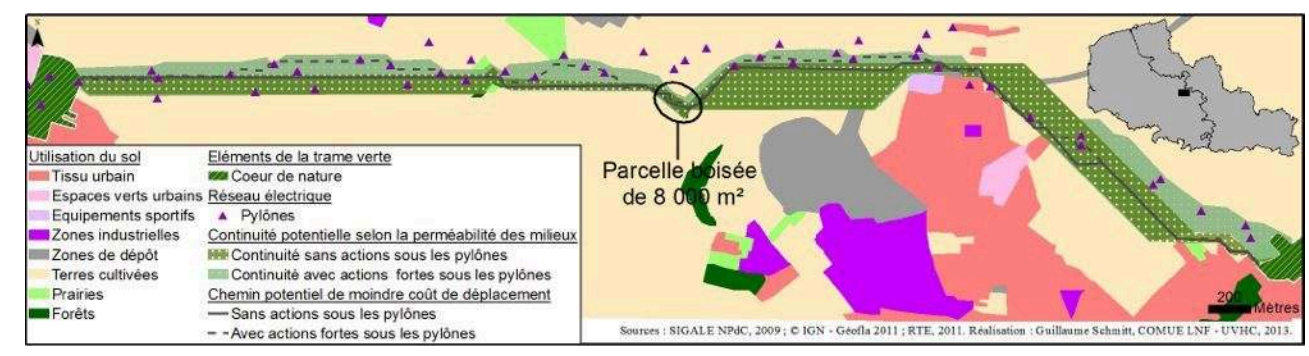
Lille, la divagation est potentiellement plus importante, le couloir de circulation gagnant par endroits une cinquantaine de mètres de largeur. La simulation de la mise en place d'habitats de substitution sous les pylônes transforme un tiers des pieds de pylônes en "espaces relais » et réoriente légèrement le chemin de moindre coût. Néanmoins, cela ne contribue pas à la réduction des effets « d'étranglement ", là où la continuité se réduit le long des zones les moins hospitalières. Nous remarquons surtout que la présence d'une parcelle boisée de quelques centaines de $\mathrm{m}^{2}$ au milieu du corridor constitue la meilleure alternative de circulation et ce, y compris après la simulation de la gestion écologique des pieds de pylônes.

Dans la modélisation réalisée à l'échelle régionale, la perméabilité générale de la mosaïque paysagère s'accentue mécaniquement sur quelque $3,2 \mathrm{~km}^{2}$ par un aménagement généralisé des emprises au sol des pylônes. Cependant, les effets potentiels sur les continuités écologiques identifiées dans la TVB semblent finalement assez réduits.

43 De manière générale, cet article montre que la proportion des espaces à caractère naturel traversés par le réseau électrique est relativement faible. Les forêts et les milieux naturels sont peu concernés alors qu'une proportion significative des terres cultivées est parcourue par les ouvrages de RTE. Les secteurs où la biodiversité est généralement qualifiée d'ordinaire sont surreprésentés, ce qui permet de considérer les emprises foncières du réseau électrique comme d'éventuelles opportunités pour agir sur la connectivité écologique dans une mosaïque paysagère fragmentée.

De manière plus précise, le croisement du réseau électrique et de la TVB montre une corrélation positive et significative et en particulier entre corridors et pylônes. La 
simulation du changement du degré de résistance des milieux situés sous les pylônes tend à démontrer que la contribution du réseau électrique à la (re)constitution de corridors « en pas japonais » est assez limitée. Seuls deux tracés de corridors identifiés dans la TVB du Nord-Pas-de-Calais connaissent un changement modéré de leur largeur ou de leur coût de déplacement.

La faible contribution du réseau électrique à l'amélioration de la connectivité pouvant s'expliquer par la faible emprise spatiale des espaces situés sous les pylônes, il serait intéressant d'analyser les effets d'une modification de l'utilisation du sol dans des secteurs où plusieurs ILT se croisent. En outre, une connaissance plus fine de l'état de la biodiversité des ouvrages de RTE permettrait de mieux évaluer comment ce réseau peut contribuer au maintien et au rétablissement de continuités écologiques pour certaines espèces.

Les différences entre la TVB définie localement en 2006 et le SRCE s'inscrivant dans un cadre national plus récent mériteraient également sans doute d'être davantage analysées, dans la mesure où la méthode de constitution a connu quelques modifications avec, par exemple, la notion de sous-trame par milieu ou bien encore l'actualisation de certains périmètres de réservoirs de biodiversité.

Enfin, l'une des interrogations portant sur la mise en place des TVB est de les mobiliser dans le cadre de mesures compensatoires (Camproux-Duffrène et Lucas, 2012). Il est également envisageable que les opérateurs, dont celui qui a la charge du réseau HT et THT, considèrent la mise en œuvre de la TVB comme un moyen de circonscrire l'urbanisation dans les pourtours de leurs infrastructures et ainsi se prémunir des effets de leur accessibilité.

\section{BIBLIOGRAPHIE}

Allag-Dhuisme, F., Barthod, C., Bielsa, S., Brouard-Masson, J., Graffin, V., Vanpeene, S. (coord), Chamouton, S., Dessarps, P.-M., Lansiart, M., Orsini A., « Prise en compte des orientations nationales pour la préservation et la remise en bon état des continuités écologiques par les grandes infrastructures linéaires de l'État et de ses établissements publics $-3^{\mathrm{e}}$ document en appui à la mise en œuvre de la Trame verte et bleue en France », Meeddm, 2010, 156 p.

Alsace Nature, « Infrastructures et continuités écologiques : étude méthodologique et application test en Alsace », rapport d'étude au ministre d'État, 2008, 134 p.

Andrews, A., « Fragmentation of habitat by roads and utility corridors: a review ", Australian Zoologist, 26 (3 \& 4), 1990, p. 130-141.

Arrif, T., Blanc, N., Clergeau, P., « Trame verte urbaine, un rapport Nature - Urbain entre géographie et écologie ", Cybergeo : European Journal of Geography, mis en ligne en décembre 2011, URL : http://cybergeo.revues.org/24862\#article-24862.

Askins, R. A, Folsom-O'Keefe, C. M., Hardy, M. C., « Effects of vegetation, corridor width and regional land use on early successional birds on powerline corridors », Plos ONE, 7(2), 2012, 10 p. 
Beier, P., Majka, D. R, Spencer, W. D., « Forks in the road: Choices in procedures for designing wildland linkages », Conservation Biology, 22, 2008, p. 836-851.

Benitez-Lopez, A., Alkemade, R., Verweij, P. A., « The impacts of roads and other infrastructure on mammal and bird populations: a meta-analysis », Biological conservation, 143, 2010,

p. 1307-1316.

Berg, A., Ahrne, K, Ockinger, E., Svensson, R., Wissman, J., « Butterflies in semi-natural pastures and power-line corridors - effects of flower richness, management, and structural vegetation characteristics ", Insect conservation and diversity, 6, 2013, p. 639-657.

Billet, P., « La prise en compte de la faune sauvage dans le cadre des procédures d'aménagement, de gestion et d'occupation de l'espace : réalités d'une apparence juridique », Natures Sciences Sociétés, 1/2006, p. 13-21.

Biotope Greet, « Analyse des potentialités écologiques du territoire régional », Diren Nord-Pasde-Calais, Région Nord-Pas-de-Calais, 2008, 66 p.

Bourget, E., Le Dû-Blayo, L, « Cartographie des paysages : apport à l'analyse des trames vertes et bleues ", Projets de paysage, $\mathrm{n}^{\circ} 4$, publié en juillet 2010, URL : http://www.projetsdepaysage.fr/fr/ cartographie_des_paysages_apport_a_l_analyse_des_trames_vertes_et_bleues\#.

Bouvier, G., « Enjeux géopolitiques autour de la distribution d'électricité en France », Hérodote, $\mathrm{n}^{\circ} 11,3 / 2003$, p. 71-87.

Broquet, T., Ray, N., Petit, E., Fryxel, J.-M., Burel, F., « Genetic isolation by distance and landscape connectivity in the American marten (Martes Americana) », Landscape Ecology, 21, 2006, p. 877-889.

Burel, F., Baudry, J., Écologie du paysage. Concepts, méthodes et applications, Cachan, Tec \& Doc, 1999, $359 \mathrm{p}$.

Calabrese, J.-M., Fagan, W.F., « A comparison-shopper's guide to connectivity metrics », Frontiers in Ecology and the Environment, 2, 2004, p. 529-536.

Caloz, R., Collet, C., Analyse spatiale de l'information géographique, Lausanne, Presses polytechniques et universitaires romandes, coll. «Ingénierie de l'environnement », 2011, 383 p.

Camproux-Duffrène, M.-P. et Lucas, M., « L'ombre portée sur l'avenir de la trame verte et bleue. Quelques réflexions juridiques ", Développement durable et territoires, vol. 3, $\mathrm{n}^{\circ} 2,2012$, URL : https://journals.openedition.org/developpementdurable/9256

CAUE Nord, « Trame verte. Politique du paysage. Un outil de gestion intégrée du paysage », conseil général du Nord, 1993, non paginé.

Cemagref, «Étude de l'intégration des continuités écologiques dans les SCoT en 2009 avant l'approbation de la loi Grenelle 2 », Meeddat, 2010, 154 p.

Charlier, B., « La défense de l'environnement : entre espace et territoire », thèse de doctorat, université de Pau et des Pays de l'Adour, 1999, 753 p.

Clarke, D.J., White, J.G., « Toward ecological management of Australian powerline corridor vegetation ", Landscape and urban planning, 86, 2008, p. 257-266.

Clauzel, C., Girardet, X., Foltête, J.-C., « Impact assessment of a high-speed railway line on species distribution: Application to the European tree frog (Hyla arborea) in Franche-Comté », Journal of environmental management, 127, 2013, p. 125-134. 
Cormier, L., Carcaud, N., « Les trames vertes : discours et/ou matérialité, quelles réalités ? ", Projets de paysage, $\mathrm{n}^{\circ}$ 2, publié en juin 2009, URL : http://www.projetsdepaysage.fr/fr/ les_trames_vertes_discours_et_ou_materialite_quelles_realites_

Decamps, H., Decamps, O., Au printemps des paysages, Paris, Buchet-Chastel, 2004, 232 p.

Denolle, A.-S., « Les études d'impact : une révision manquée ? », Revue française de droit constitutionnel, $\mathrm{n}^{\circ}$ 87, 3/2011, p. 499-514.

Diren Nord-Pas-de-Calais, « Schéma de services collectifs des espaces naturels et ruraux. Contribution de la Région Nord-Pas-de-Calais », ministère de l'Agriculture, de la Pêche et de l'Alimentation, 1999, $152 \mathrm{p}$.

EDF et GDF, « Modalité de gestion de la végétation sous et aux abords des lignes électriques », $2000,60 \mathrm{p}$.

Fahrig, L., «Effect of habitat fragmentation on biodiversity », Annual Review of Ecology, 34, 2003, p. 487-515.

Foltête, J.-C., « Paysage et Mouvement. De l'écologie aux déplacements urbains : éléments pour une identification des paysages préférentiels ", volume II, habilitation à diriger des recherches, université de Franche-Comté, 2006, 223 p.

Forman, R., Godron, M., Landscape ecology, Haboken, Wiley and Sons, 1986, 640 p.

Hendoux, F., « Atlas régional de la trame verte et bleue. Cahier méthodologique », Centre régional de phytosociologie/Conservatoire botanique national de Bailleul, 2005, 73 p.

Hirzel, A.H., Helfer, V., Metral, F., « Assessing habitat-suitability models with a virtual species », Ecological Modelling, $\mathrm{n}^{\circ}$ 145, 2001, p. 111-121.

Jaeger, J.A.G., Fahrig, L., « Effects of road fencing on population persistence », Conservation Biology, 18, 2004, p. 1651-1657.

Kabouche, B., Bayeul, J., Zimmermann, L., Bayle, P., « La mortalité des oiseaux sur le réseau électrique aérien : enjeux et perspectives en Paca », Dreal Paca, 2006, 108 p.

Kindlmann, P., Burel, F., « Connectivity measures: a review », Landscape ecology, 23, 2008, p. 879-890.

Konga, F., Yinb, H., Nakagoshic, N., Zongb, Y., « Urban green space network development for biodiversity conservation: Identification based on graph theory and gravity modeling ", Landscape and Urban Planning, 95, 2010, p. 16-27.

Labussière, 0. , « Les stratégies esthétiques dans la contestation des projets d'aménagement : le milieu géographique entre singularité et exception ", L'Information géographique, vol. 73, 2/2009, p. 68-88.

Le Floch, S., Devanne, A.-S., Deffontaines, J.-P., « Fermeture du paysage : au-delà du phénomène, petite chronique d'une construction sociale ", L'Espace géographique, t. 34, 1/2005, p. 49-64.

Liénard, S., Clergeau, P., « Trame verte et bleue : utilisation des cartes d'occupation du sol pour une première approche qualitative de la biodiversité ", Cybergeo : European Journal of Geography, mis en ligne en mars 2011, URL : URL : http://cybergeo.revues.org/23494.

Medd et Ademe, « Guide de l'étude d'impact sur l'environnement d'un parc éolien », 2005, 123 p. Meehan, A. L., Haass, C.A., « Use of a powerline corridor by breeding and wintering birds in Giles County », Virginia Journal of Science, 48(4), 1997, p. 259-264. 
Morelli, F., Beim, M., Jerzak, L., Jones, D., Tryjanowski, P., « Can roads, railways and related structures have positive effects on birds? - A review ", Transportation Research Part D, 30, 2014, p. 21-31.

Préfet du Nord-Pas-de-Calais et Région Nord-Pas-de-Calais, Schéma régional de cohérence écologique - Trame verte et bleue du Nord-Pas-de)Calais, document du travail, 2012a, 315 p.

Préfet du Nord-Pas-de-Calais et Région Nord-Pas-de-Calais, Schéma régional de cohérence écologique - Trame verte et bleue du Nord-Pas-de-Calais, cahier technique, document du travail, 2012b, 148 p.

Région Nord-Pas-de-Calais, Schéma régional d'aménagement et de développement du territoire, 2006, 119 p.

RTE et AMF, « Convention de partenariat entre l'Association des maires de France et RTE », 2008, $6 \mathrm{p}$.

RTE et ANEM, « Convention de partenariat entre l'association nationale des élus de la montagne et RTE », 2013, 6 p.

RTE, « Protocoles. Passage des lignes électriques. Dommages permanents. Dommages instantanés ", 2005, 34 p.

RTE et FNC, «Convention national de partenariat. Aménagement et exploitation de terrains situés dans l'emprise des lignes électriques haute et très haute tension », 2008, 30 p.

Rubolini, D., Gustin, M., Bogliani, G., Garavaglia, R., « Birds and powerlines in Italy : an assessment », Bird Conservation International, 15, 2005, p. 131-145.

Sainteny, G., Salles, J.-M., Duboucher, P., Ducos, G., Marcus, V., Paul, E., Auverlot, D., Pujaol, J.-L., Les Aides publiques dommageables à la biodiversité, Paris, La Documentation française, 2012, 414 p.

Spellerberg, I.F., " Ecological effects of roads and traffic: a literature review », Global Ecology and Biogeography Letters, 7, 1998, p. 317-333.

Valet, J.-M., « Des lieux que les botanistes n'ont pas l'habitude de visiter », Reso Nord-Est, $\mathrm{n}^{\circ} 7$, RTE, 2011, p. 3.

Van der Zande, A.N., Ter Keurs, W.J., Van der Weijden, W.J., « The impact of roads on the densities of four bird species in an open field habitat - Evidence of long distance effect ", Biological Conservation, 18, 1980, p. 299-321.

Vandevelde, J.-C., « Les choix de tracé des grandes infrastructures de transport : quelle place pour la biodiversité ? ", Développement durable et territoires, vol. 4, n 1, 2013, URL : https:// journals.openedition.org/developpementdurable/9721

Vanpeene-Bruhier, S., Pissard, P.-A. et Kopf, M., « Prise en compte de la biodiversité dans les projets d'aménagement : comment améliorer la commande des études environnementales?", Développement durable et territoires, vol. 4, $\mathrm{n}^{\circ}$ 1, 2013, https://journals.openedition.org/ developpementdurable/9701

Vimal, R., Mathevet, R., « La carte et le territoire : le réseau écologique à l'épreuve de l'assemblée cartographique », Cybergeo : European Journal of Geography, mis en ligne en décembre 2011, URL : http://cybergeo.revues.org/24841. 


\section{NOTES}

1. Routes, voies ferrées, canaux, transport d'énergie...

2. Art. 121 de la loi ${ }^{\circ} 2010-788$ du 12 juillet 2010.

3. RTE est l'opérateur du réseau français de transport haute et très haute tension d'électricité.

4. "La biodiversité en réseaux: de la décision aux outils ou comment mailler le territoire? (2010-2013)».

5. Le schéma régional de cohérence écologique (SRCE), bien qu'avancé, est en cours de finalisation. Suite à la consultation des collectivités, du conseil scientifique régional du patrimoine naturel et de l'enquête publique, plusieurs modifications ont été apportées et un nouveau projet SRCE-TVB a été proposé en avril 2014. Il n'est pas adopté à l'heure actuelle. Les diagnostics et descriptifs attenants ont toutefois été mobilisés.

6. ERDF, GRTgaz, RFF, RTE, TIGF, VINCI autoroutes et VNF.

7. L'article 23 précise que les SSCENR identifient « les réseaux écologiques, les continuités et les extensions des espaces protégés qu'il convient d'organiser » (loi n 99-533 du 25 juin 1999).

8. Indicateur de fragmentation des espaces naturels développé par l'Irstea en 2010, IRL : https:// www.cohesion-territoires.gouv.fr/

9. Au sens de l'écologie du paysage (Burel et Baudry, 1999).

10. Région, parc naturel régional, établissement public de coopération intercommunale et commune.

11. L'annonce d'une construction peut susciter des conflits entre habitants, usagers, acteurs publics et parapublics durant lesquels l'argument esthétique est mobilisé (Charlier, 1999; Labussière, 2009).

12. Les PLU de la communauté urbaine de Lille et de la communauté d'agglomération de Maubeuge ont été consultés du fait de la disponibilité de l'information et des contrastes d'utilisation du sol.

13. Les servitudes de passage ne sont pas prises en compte car elles sont peu contraignantes (loi $\mathrm{n}^{\circ}$ 2000-108 du 10 février 2000 et art. R. 126-1 du code de l'urbanisme). La largeur des servitudes varie selon le voltage, l'enfouissement ou nom des lignes... (arrêté technique du 17 mai 2001 fixant les conditions techniques auxquelles doivent satisfaire les distributions d'énergie électrique).

14. Cartographes et ingénieurs de RTE.

15. $7,2 \%$ du réseau RTE passe par des zones industrielles ou commerciales, alors que ces dernières n'occupent que $5,2 \%$ du Nord-Pas-de-Calais.

16. «RTE : biodiversité sous les pylônes et les lignes THT », unité de recherche Efno.

17. « Emprise des pylônes et biodiversité », Conservatoire botanique national de Bailleul.

18. Pour éviter d'éventuelles confusions, les termes de 2006 sont employés.

\section{RÉSUMÉS}

Depuis peu, les infrastructures linéaires de transport (ILT) ne sont plus principalement considérées comme des obstacles à la connectivité écologique, mais aussi comme des opportunités foncières potentielles pour la création ou le maintien de milieux favorables à la biodiversité dans le cadre de la mise en place de la Trame verte et bleue (TVB). Au sein d'un paysage très fragmenté, les ILT, à l'instar des lignes électriques haute et très haute tension, 
peuvent-ils offrir des habitats favorisant le déplacement de certaines espèces ? Cet article cherche à identifier les points de contact entre le réseau électrique et la TVB du Nord-Pas-deCalais à partir des méthodes de l'écologie du paysage. Les bases de données créées ont été appliquées à la mise en évidence du niveau de recouvrement entre le réseau électrique et la TVB ; à l'évaluation de l'utilisation du sol affectée par les ouvrages, et de la covisibilité de ces derniers ; à l'estimation, par simulation, des effets d'une modification de l'utilisation du sol sur la connectivité écologique et le paysage.

Recently, linear utility infrastructures stopped being considered principally as obstacles to ecological connectivity and have started to be considered as potential land use opportunities for the creation and maintenance of environments conducive to biodiversity within the framework of the Green Blue Networks initiative (in French, Trame verte et bleue or TVB). In a fragmented landscape, can linear utility infrastructures, in the same way as high and low voltage power lines, offer habitats favourable to the movements of certain species? This article seeks to identify the points of contact between the electric grid and the Green Blue Network of the Nord-Pas de Calais region based on landscape ecology methods. The data-bases created were used to: show the overlap between the electric grid and the green blue network; the evaluation of how land use is affected by the works, as well as their co-visibility; and the estimation by simulation of the effects of a change in land use on ecological connectivity and the landscape.

\section{INDEX}

Mots-clés : paysage, biodiversité, continuité écologique, réseau électrique, Trame verte et bleue, écologie du paysage

Keywords : landscape, biodiversity, ecological continuity, electric grid, green and blue network, landscape ecology

\section{AUTEURS}

\section{GUILLAUME SCHMITT}

Guillaume Schmitt est maître de conférences en géographie, CALHISTE, université de Valenciennes et du Hainaut-Cambrésis.

schmitt_guillaume[at]yahoo[dot]fr

\section{MAGALIE FRANCHOMME}

Magalie Franchomme est maître de conférences en géographie, TVES, université Lille 1. magalie.franchomme[at]univ-lille1[dot]fr

\section{CHRISTELLE HINNEWINKEL}

Christelle Hinnewinkel est maître de conférences en géographie, TVES, université Lille 1. christelle.hinnewinkel[at]univ-lille1[dot]fr 